Ann. Zootech., I975, 24 (I), r-I2.

\section{I.N.R.A. \\ BIBLIOTHEQUE UO 35906 \\ DOMAINE DE CROUELLE \\ CLERMONT-FD CEDEX 2}

\title{
DÉTERMINATION DU BESOIN ÉNERGÉTIQUE D'ENTRETIEN DU PORC
}

\author{
A. M. LEROY \\ Professeur honoraire de Zootechnie à l'Institut national agronomique, \\ 62, rue du Château, \\ 95340 Saint Leu la Forêt
}

RÉSUMÉ

Il existe dans la bibliographie plusieurs formules permettant la détermination du besoin d'entretien en fonction du poids vif. Ces formules expriment le besoin, soit en énergie métabolisable, soit en énergie nette. Pour comparer aisément les résultats obtenus par ces deux modes de détermination, il suffit de se rappeler, avec Kielanowski, que, chez les porcs normalement alimentés, le besoin d'énergie nette est de l'ordre de 72 p. Ioo de l'énergie métabolisable.

Les résultats obtenus au moyen de 5 formules différentes, évalués en énergie nette, ont été comparés, pour des porcs de Io à $120 \mathrm{~kg}$. Ils présentent entre eux de fortes différences. A l'aide de nombreuses données de bilans énergétiques trouvées dans les publications des stations expérimentales danoises, de I956 à I970, et connaissant les poids des animaux ainsi que les apports d'énergie métabolisable, nous avons précisé les acquisitions d'énergie correspondant aux accroissements quotidiens de poids, les quantités de chaleur émises et les quantités correspondantes de matière sèche consommées. Il est alors possible d'obtenir dans chaque cas la valeur $\mathrm{E}$ du besoin d'entretien, en appliquant la relation : Énergie métabolisable $(M)=$ Énergie d'entretien (E) - Énergie de croissance (P) + MS $\times$ I kcal, dans laquelle MS représente la quantité de matière sèche ingérée en grammes. On admet dans ce cas que le rapport $\frac{\mathrm{M}-(\mathrm{F}+\mathrm{P})}{\mathrm{MS}}$ est égal à l'unité.

Les résultats de 164 expériences, effectuées sur 36 porcs entre 20 et I oo $\mathrm{kg}$ par LUDVIGSEx et THORBEK, d'une part (groupe A) et par Nielsen, d'autre part (groupe B) ont permis, à partir de valeurs moyennes de $\mathrm{E}$ calculées séparément pour chaque groupe, de construire des graphiques faisant connaître dans les deux cas, la variation de $E$ (en ordonnées) par rapport au poids (en abscisses). On obtient ainsi deux courbes, sensiblement parallèles, séparées par une distance équivalente à r 50 kcal. Pour comprendre l'existence de cet écart, il suffit de constater que les rations distribuées par Nielsen sont moins digestibles que celles de Ludvigsen et Thorbek, soit 3,65 kcal d'énergie métabolisable par g contre 4,r. Pour assurer la même ration d'entretien, les porcs du groupe $B$ devaient donc consommer davantage de matière sèche que ceux du groupe $A$ Pour des porcs de $50 \mathrm{~kg}$, par exemple, la différence de $150 \mathrm{kcal}$ entre les valeurs trouvées pour IE correspondaient à une différence dans la consommation de matière sèche de I 50 g. Cette constatation fournit une excellente preuve de la règle qui lie l'énergie calorifique dépensée en supplément du métabolisme de jeûne à la consommation de matière sèche. 


\section{INTRODUCTION}

Dans les comptes rendus des travaux publiés à l'occasion des symposiums sur l'énergie métabolique des animaux de ferme, publiés par la Fédération Européenne de Zootechnie, le lecteur peut trouver diverses formules qui permettent de calculer, en fonction du poids $\mathrm{P}$ d'un porc, le besoin d'énergie d'entretien correspondant $\mathrm{F}$. Ce besoin s'exprime, selon les formules utilisées, soit en quantités d'énergie métabolisable, exprimées en kilocalories, soit en quantités d'énergie nette. Pour comparer entre eux les résultats obtenus à l'aide de ces diverses formules, il suffit de se souvenir, comme le rappelle KIELANOWSKI, que le rapport de l'énergie nette à l'énergie métabolisable pour des porcs alimentés normalement est de l'ordre de 72 p. I00.

Nous avons retenu cinq de ces formules proposées par BREIREM, KoTARBINSKA, HoFFmanN, Thorbek et KiElanowski.

L'une d'entre elles se présente sous la forme d'une équation du premier degré $a+b \mathrm{P}$, dans laquelle $\mathrm{P}$ représente le poids de l'animal. Pour les autres, $\mathrm{P}$ se trouve affecté d'un exposant, qui est, soit 0,56 , soit 0,75 . Nous savons que l'exposant 0,75 est couramment utilisé d'un commun accord entre les spécialistes, pour la détermination du poids métabolique des animaux, ce qui permet d'utiles comparaisons entre les besoins énergétiques de sujets appartenant aux diverses espèces d'homéothermes. Mais le coefficient 0,56 , proposé à la suite de nombreuses expériences par notre collègue BREIREM, peut se justifier pour l'espèce porcine, pour diverses raisons. La première tient à la morphologie particulière du porc, qui possède une forme arrondie dans son ensemble, ce qui réduit davantage que chez les ruminants la surface corporelle exposée au milieu extérieur par rapport au poids vif. La seconde nous semble en rapport avec l'existence d'une couche de matière grasse superficielle, progressivement croissante avec l'âge de l'animal, qui joue le rôle d'un écran efficace entre les sources de chaleur internes et l'air environnant. Ces circonstances peuvent fort bien modifier la nature et l'intensité des échanges thermiques entre l'animal et ce qui l'entoure. Les cinq formules dont nous nous proposons de comparer les résultats d'application sont rapportées dans le tableau $\mathrm{I}$.

TABLEAU I

Formules proposées pour l'évaluation des besoins $E$ d'entretien des porcs

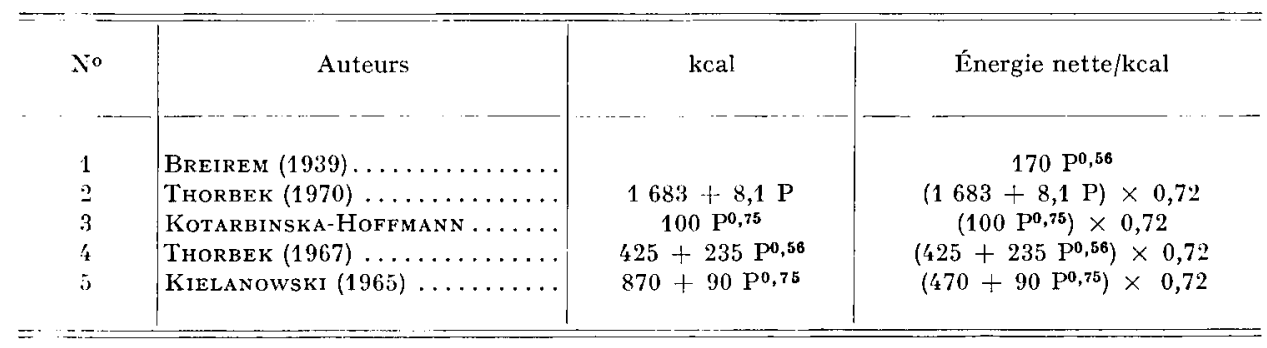


Nous avons calculé, pour des porcs dont le poids varie de ro à I20 $\mathrm{kg}$, les résultats obtenus par l'application de ces diverses formules (tabl. 2). On constate aisément qu'il existe entre ces données de profondes discordances pour les poids les plus faibles et les plus élevés. On peut ainsi remarquer que la progression des valeurs de $\mathrm{E}$, avec la première formule, suit d'assez près les résultats moyens indiqués par la colonne 6 . Il est donc nécessaire de rechercher une autre méthode, puisque celles que nous venons d'examiner ne donnent pas entièrement satisfaction. C'est surtout la formule 2 (ThorbeK) qui diffère le plus des 4 autres. De plus, les formules 4 et 5 son les plus semblables.

TABLEAU 2

Besoins d'entrotien, évalués en $\mathrm{kg}$ d'énergie nette

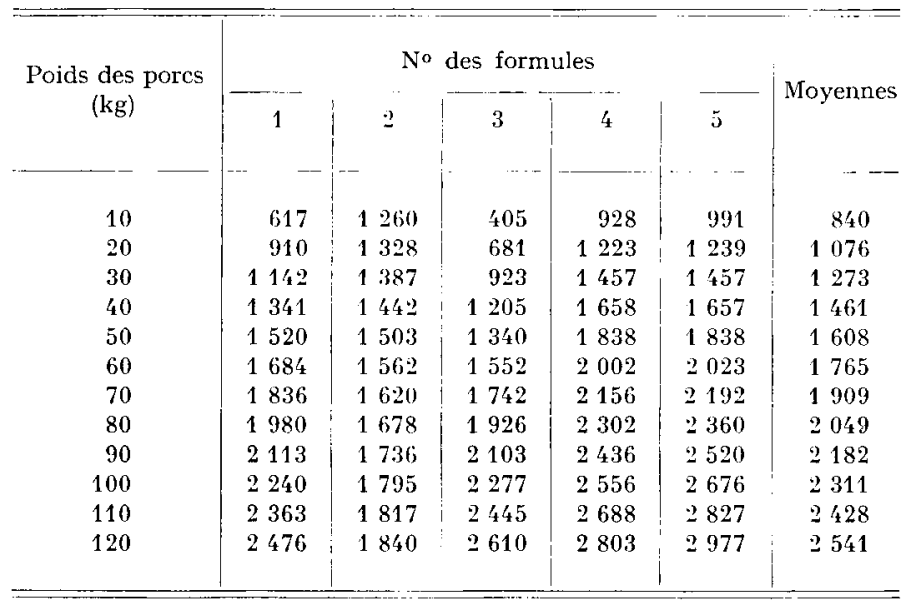

Depuis I949, nous avons tenté de prouver, dans de nombreuses publications que les conditions d'équilibre énergétique d'un animal normalement alimenté pouvaient s'exprimer de la manière suivante :

$$
\left(\begin{array}{c}
\text { Énergie } \\
\text { métabolisable }
\end{array}\right)=\left(\begin{array}{c}
\text { Énergie } \\
\text { d'entretien }
\end{array}\right)+\left(\begin{array}{c}
\text { Energie de } \\
\text { la production }
\end{array}\right)+\mathrm{MS} \times k
$$

dans laquelle MS est la quantité de matière sèchée ingrée, exprimée en grammes. L'expérience nous a montré que, pour des espèces aussi différentes que les animaux de basse-cour, les porcs et les ruminants, le nombre de kilocalories correspondant à l'expression $\mathrm{K}$ est voisin de l'unité et peut pratiquement se confondre avec elle.

Bien entendu, cette formule ne s'applique qu'à la condition que l'animal soit placé dans des conditions d'environnement ne mettant en jeu aucun mécanisme de défense, soit contre le froid, soit contre l'excès de chaleur.

De l'équation d'équilibre précédente, nous pouvons obtenir la valeur de $\mathrm{K}$ de la manière suivante :

$$
\mathrm{K}=\frac{\mathrm{M}-(\mathrm{E}+\mathrm{P})}{\mathrm{MS}}
$$

expression dans laquelle M est l'apport d'énergie métabolisable, E le besoin d'entre- 
tien, P l'apport d'énergie représenté par les gains quotidiens de matières azotées et grasses, et MS la masse de la matière sèche consommée.

En utilisant les données numériques provenant d'une part de 96 expériences publiées en 1956 par LUDVIGSEN et THORBEK, et d'autre part, de I2 expériences publiées en 1967 par THORBEK, nous avons obtenu, en prenant comme valeurs de $\mathrm{E}$ celles de la formule de BREIREM (tabl. 2, col. r), les résultats rapportés au tableau 3.

TABLEAU 3

Détermination du coefficient $K$ pour 2 séries d'expériences

\begin{tabular}{|c|c|c|c|}
\hline \multicolumn{2}{|c|}{$\begin{array}{c}1 \\
\text { Moyennes pour } 96 \text { expériences } \\
\text { (LudVIGSEN-THORBEK, 1956) }\end{array}$} & \multicolumn{2}{|c|}{$\begin{array}{c}2 \\
\text { Moyennes pour 12 expériences } \\
\text { (THORBEK, 1967) }\end{array}$} \\
\hline $\begin{array}{l}\text { Poids des porcs } \\
\qquad(\mathrm{kg})\end{array}$ & $\begin{array}{l}\text { Valeurs de K } \\
\text { (kcal) }\end{array}$ & $\begin{array}{l}\text { Poids des porcs } \\
\qquad(\mathrm{kg})\end{array}$ & $\begin{array}{l}\text { Valeurs de } \mathrm{K} \\
\text { (kcal) }\end{array}$ \\
\hline \multicolumn{4}{|l|}{$\ldots$} \\
\hline 26,3 & 1,18 & 29,3 & 1,43 \\
\hline 36,6 & $0,9 \mathbf{q}_{\mathbf{k}}$ & 35,7 & 1,23 \\
\hline 48,5 & 0,87 & 43,6 & 1,20 \\
\hline 62,2 & 0,89 & 52,3 & 1,04 \\
\hline 75,2 & 0,80 & 61,4 & 0,97 \\
\hline 90,7 & 0,83 & 71,9 & 0,95 \\
\hline & & 83,1 & 0,92 \\
\hline \multicolumn{4}{|c|}{ Moyenne générale pondérée 0,99} \\
\hline
\end{tabular}

Nous constatons ainsi que les valeurs de $\mathrm{K}$ tendent à décroître, à mesure que les animaux gagnent du poids. Pour expliquer ce fait, nous avons pensé que les besoins d'entretien évalués d'après BREIREM pouvaient se trouver erronés, particulièrement pour les animaux de faible poids. Cette hypothèse se trouve en quelque sorte confirmée, d'une part, par une publication récente de TroRbEr. Cet auteur a mesuré directement sur des porcs de $\mathrm{I} 6,4 \mathrm{~kg}$ en état de jeûne placés dans un calorimètre, un besoin d'entretien exprimé en énergie métabolisable de I $800 \mathrm{kcal}$, correspondant à I $296 \mathrm{kcal}$ d'énergie nette. D'autre part, en reprenant dans une de nos publications datée de 1946 des résultats de bilans énergétiques obtenus sur des porcelets élevés sans mère, nous avons trouvé pour des animaux de $10,5 \mathrm{~kg}$ un besoin d'entretien exprimé en énergie nette de $970 \mathrm{kcal}$. En partant de ces constatations, nous avons tenté d'utiliser une méthode de détermination empirique du besoin $\mathrm{E}$, en nous servant exclusivement de bilans expérimentaux obtenus par calorimétrie indirecte.

Pour chaque expérience effectuée sur un animal de poids donné, on a déterminé la quantité journalière $\mathrm{M}$ d'énergie métabolisable, la quantité MS correspondante de matière sèche ingérée, et la somme $P$ de l'énergie accumulée pendant le même temps sous forme de gains corporels de matières azotées et grasses. On peut alors obtenir la valeur $\mathrm{E}$ du besoin d'entretien, en prenant une valeur de $\mathrm{K}$ égale à l'unité, par le calcul ci-après :

$$
\mathrm{E}=\mathrm{M}-(\mathrm{P}+\mathrm{MS})
$$


Voici deux exemples de cas, calculs empruntés l'un aux travaux de L,UDVIGSENThorbek, et l'autre aux travaux de NiEL,SEN (I970) (tabl. 4).

\section{TABLEAU 4}

Exemples de calculs du besoin E d'entretien

\begin{tabular}{|c|c|c|c|c|c|}
\hline Auteurs & $\begin{array}{l}\text { Poids des } \\
\text { porcs } \\
(\mathrm{kg})\end{array}$ & $\begin{array}{c}\text { Énergie } \\
\text { métabolisable } \\
\text { (kcal) }\end{array}$ & $\begin{array}{c}\text { Énergie à } \\
\text { la production } \\
(\text { kcal })\end{array}$ & $\begin{array}{c}\text { Matière sèche } \\
\text { consommée } \\
\times \text { kcal }\end{array}$ & $\begin{array}{c}\text { Besoin } \\
\text { d'entretien } \\
\text { E }\end{array}$ \\
\hline$-\cdots-$ & . - - - & - & $-\cdots$ & $\ldots-$ & - \\
\hline $\begin{array}{l}\text { LUdVigsen-Thorbek }(1956) \\
\text { NiELSEN }(1970) \ldots \ldots \ldots \ldots\end{array}$ & $\begin{array}{l}44,0 \\
56,0\end{array}$ & $\begin{array}{l}4933 \\
5862\end{array}$ & $\begin{array}{l}1963 \\
2537\end{array}$ & $\begin{array}{l}1197 \\
1518\end{array}$ & $\begin{array}{l}1773 \\
1807\end{array}$ \\
\hline
\end{tabular}

En utilisant les données provenant de I64 bilans énergétiques, nous avons obtenu ainsi, pour des porcs dont le poids variait entre 22 et $95 \mathrm{~kg}$, I64 valeurs de $\mathrm{E}$, que nous avons divisé en deux groupes, le premier constitué avec les résultats de LUDVIGSEN-THORBEK et le second, avec ceux de NIELSEN. Nous avons rapproché les résultats obtenus pour des animaux de poids voisins, et calculé ainsi les moyennes de chaque groupe de poids et celles des valeurs de $\mathrm{E}$ qui leur correspondaient. Enfin, en pondérant les résultats observés sur chaque groupe, pour tenir compte des différences d'effectif entre les groupes, nous avons dressé un tableau représentant 1'ensemble de toutes les données utilisées (tabl. 5).

\section{TABLEAU 5}

Variation $d u$ besoin $E$ en fonction du poids des porcs

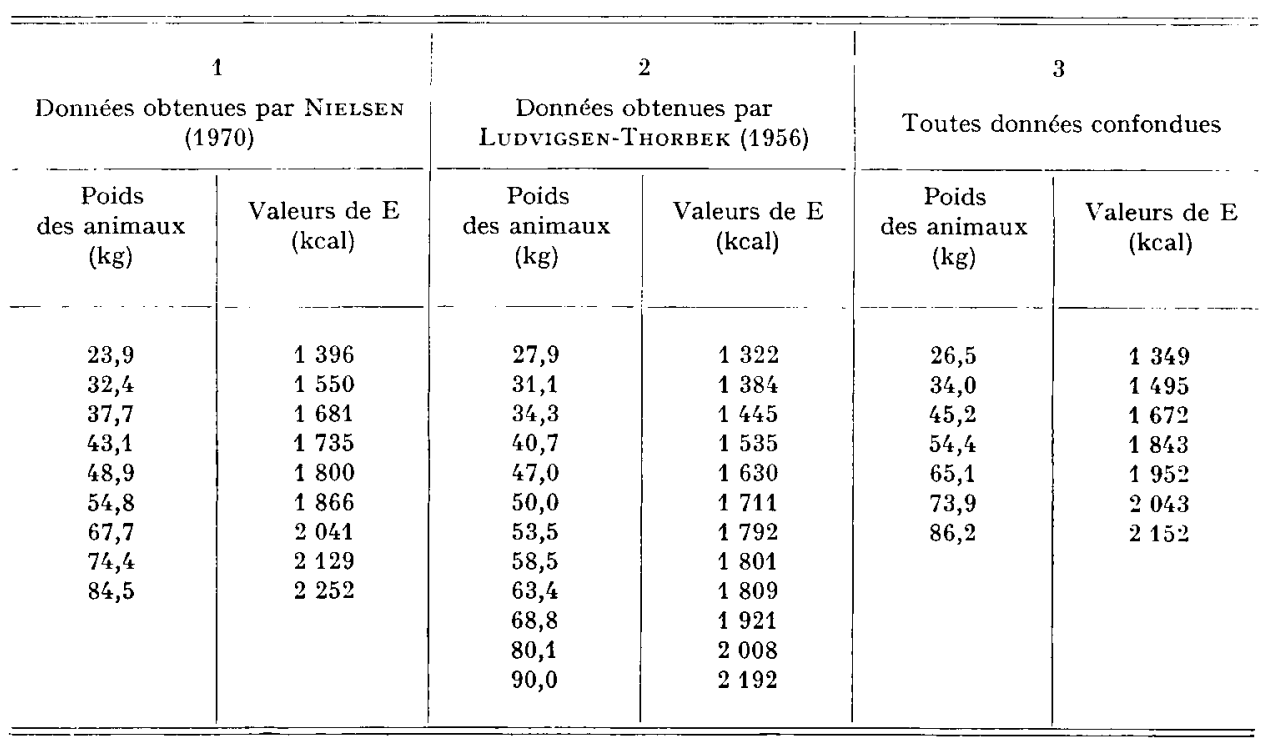


Pour mieux comparer ces trois séries de données, nous avons construit un graphique, en représentant chaque couple poids-valeur de E par un point. Les poids ont été ainsi portés en abscisses, et les valeurs de $\mathrm{E}$ en ordonnées. Le même signe est utilisé pour les couples d'une même série (fig. I).

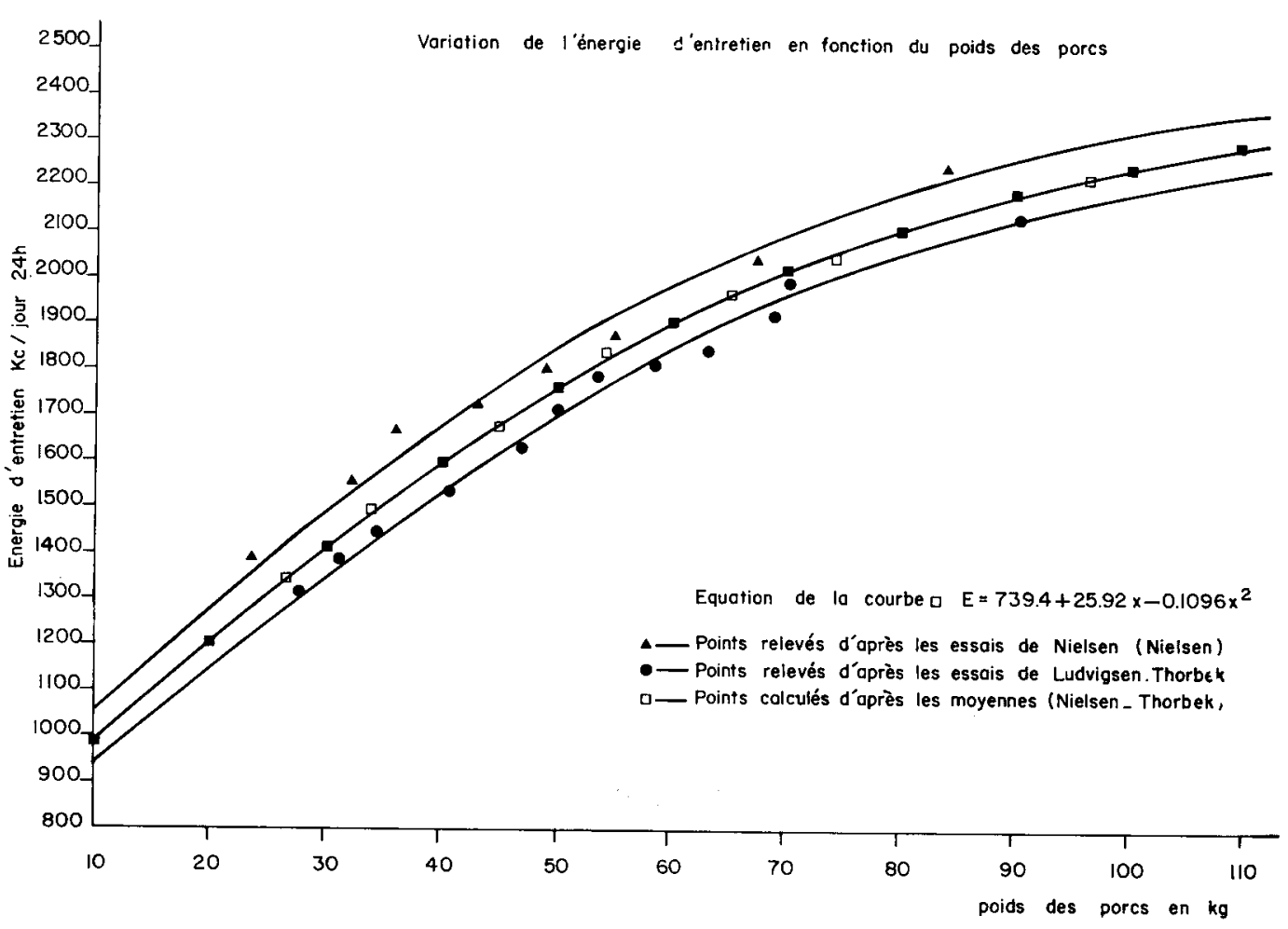

FIG. I

On voit ainsi que chaque groupe de points s'inscrit sur une courbe relativement régulière. Nous obtenons de la sorte trois courbes superposées, sensiblement parallèles. Celle du bas correspond aux couples LudviGsen-THORBEK, la courbe médiane représente les moyennes et la courbe supérieure a été construite avec les données de NIELSEN. On remarquera que cette dernière est plus éloignée de la médiane que 1'autre. Nous verrons plus loin ce qu'il faut penser de cette observation.

Frappés par la parfaite régularité de la courbe médiane, nous avons cherché si elle ne correspondait pas aux paramètres d'une fonction relativement simple. Nous avons ainsi obtenu une équation du deuxième degré de la forme $\mathrm{E}=739,40+25,92 \mathrm{P}-0, \operatorname{Iog} 6 \mathrm{P}^{2}$.

En résolvant cette équation pour $\mathrm{P}=\mathrm{I}$ oo, nous obtenons pour résultat $2325 \mathrm{kcal}$ nombre qui est très voisin de celui de $2240 \mathrm{kcal}$ proposé comme valeur du besoin d'entretien d'un porc de IOo $\mathrm{kg}$ par BREIREM. 


\section{DISCUSSION}

Il reste maintenant à expliquer pour quelle raison la consultation de ce graphique fournit des valeurs de Ei différentes pour la même valeur de P. Pour cela, en examinant avec attention les compositions des rations distribuées aux animaux des deux groupes, il est aisé de constater qu'elles sont différentes par leur digestibilité. En fait, les rations composées par NIELSEN sont moins digestibles que celles de LUDVIGSEN-THORBEK et possèdent pour cette raison, à égalité de poids de matière sèche, une valeur alimentaire plus faible. Tandis que les premières apportent en moyenne, pour un gramme de matière sèche, 3,65 kilocalories d'énergie métabolisable, les secondes en fournissent 4 , I. Un calcul simple permettrait, d'autre part, d'évaluer l'apport d'un gramme de matière sèche des rations hypothétiques auxquelles correspond la courbe moyenne correspondant à 3,9 kilocalories/gramme.

Pour 3,65 kcal d'énergie métabolisable de la ration moyenne NIELSEN, l'apport d'énergie nette est, d'après nos conventions, de $2,65 \mathrm{kcal}$. L,e rendement en énergie nette d'un tel aliment est donc de $\frac{3,65}{2,65} \times$ Iоo $=72,6$ p. IоO.

D'autre part, pour $4 \mathrm{kcal}$, de la ration I,UDVIGSEN-THORBEK, la quantité correspondante d'énergie nette est de $\frac{4, \mathrm{IO}}{3, \mathrm{II}} \times$ IoO, c'est-à-dire $75,8 \mathrm{p}$. IOO.

Nous constatons ainsi que le rendement en énergie nette de l'énergie métabolisable, fixé a priori par KIEIANOWSKI à 72 p. IoO, peut fortement varier arec la digestibilité des rations distribuées, dans des limites comprises entre 69,0 p.Ioo pour une alimentation exagérément chargée en matériaux peu digestibles et 77,8 p. Ioo pour un régime d'une digestibilité de $95 \mathrm{p}$. Ioo exclusivement composé de lait de truie.

Considérons par exemple un porc de $50 \mathrm{~kg}$. S'il appartient à la série NIELSEN il lui faudra, pour équilibrer ses dépenses d'entretien, I $850 \mathrm{kcal}$. d'énergie nette. La satisfaction de ce besoin, évaluée en énergie métabolisable sera de $\frac{I 850 \times \text { IOo }}{72,6}=2548,2 \mathrm{kcal}$; il faudra donc pour cela une consommation de matière sèche de $\frac{2548,2}{3,65}=698, \mathrm{I}$ g.

Effectuons des calculs analogues pour un porc de la série L,UDVIGSEN-THORBEK, qui a besoin de I $700 \mathrm{kcal}$ d'énergie nette, correspondant à $\frac{I 700 \times \text { IOO }}{75,8}=2246,6 \mathrm{kcal}$ d'énergie métabolisable, lequel sera satisfait par une consommation de $\frac{2246,6}{4, \mathbf{I}}=547 \mathrm{~g}$ de matière sèche.

Nous voyons ainsi qu'à la différence entre les besoins d'entretien de I $850-\mathrm{I} 700=\mathrm{I}_{50} \mathrm{kcal}$ correspond une différence de consommation de matière sèche de $698-547=\mathrm{r} 5 \mathrm{I} \mathrm{g}$. Nous retrouvons donc bien ainsi la quantité d'énergie entraînée par la consommation d'un gramme de matière sèche, que nous avons évaluée à I kilocalorie.

Il est donc ni logique, ni raisonnable, de se baser pour le calcul des rations à 
distribuer aux porcs exclusivement sur l'apport d'énergie métabolisable, puisque le rendement de cette dernière en énergie nette est fortement influencé par le coefficient de digestibilité des aliments à distribuer. Il faut donc évaluer correctement en énergie nette les besoins des porcs, et de chercher ensuite à satisfaire ces besoins par des apports d'énergie métabolisable provenant d'un rationnement dont on connaît à l'avance la digestibilité.

\section{CONCLUSION}

Pour l'évaluation correcte des besoins d'entretien des porcs, nous proposons de prendre pour base les indications de la courbe médiane du graphique, parce que les mélanges alimentaires à base de céréales et de compléments azotés distribués aux porcs dans les conditions courantes de la pratique sont caractérisés ordinairement par un apport d'énergie métabolisable voisin de $3,9 \mathrm{kcal} / \mathrm{gramme}$ de matière sèche. Traduites sous forme de tableau, les données obtenues à partir de cette courbe sont consignées dans le tableau 6 .

TABI,EAU 6

Besoins d'entretien des porcs évalués en énergie nette

\begin{tabular}{c|c}
\hline $\begin{array}{c}\text { Poids des porcs } \\
(\mathrm{kg})\end{array}$ & $\begin{array}{c}\text { Valeurs correspondantes de } \mathrm{E} \\
\text { (kcal) }\end{array}$ \\
\hline & \\
10 & 995 \\
20 & 1210 \\
30 & 1410 \\
40 & 1600 \\
50 & 1760 \\
60 & 1900 \\
70 & 2010 \\
80 & 2100 \\
90 & 2190 \\
100 & 2240 \\
110 & 2270 \\
120 & 2290 \\
\hline
\end{tabular}

Lorsque les rations que 1'on désire utiliser diffèrent, en plus ou en moins, de 3,9 kcal d'énergie métabolisable par gramme de matière sèche, il faut apporter aux valeurs de $\mathrm{E}$ figurant dans le tableau précédent certaines corrections. De telles circonstances se produisent, soit lorsque 1'on fait entrer dans les mélanges alimentaires du son de froment, de la pulpe de betterave, du marc de pomme ou de la luzerne déshydratée, ce qui décroît le coefficient de digestibilité, soit lorsque les rations comportent du suif ou du saindoux, ce qui donne au contraire, des quantités d'énergie métabolisable par $\mathrm{g}$ de MS supérieures à 3,9 $\mathrm{kcal}$. Afin de tenir compte des corrections nécessaires lorsque les rations possèdent des digestibilités nettement différentes 


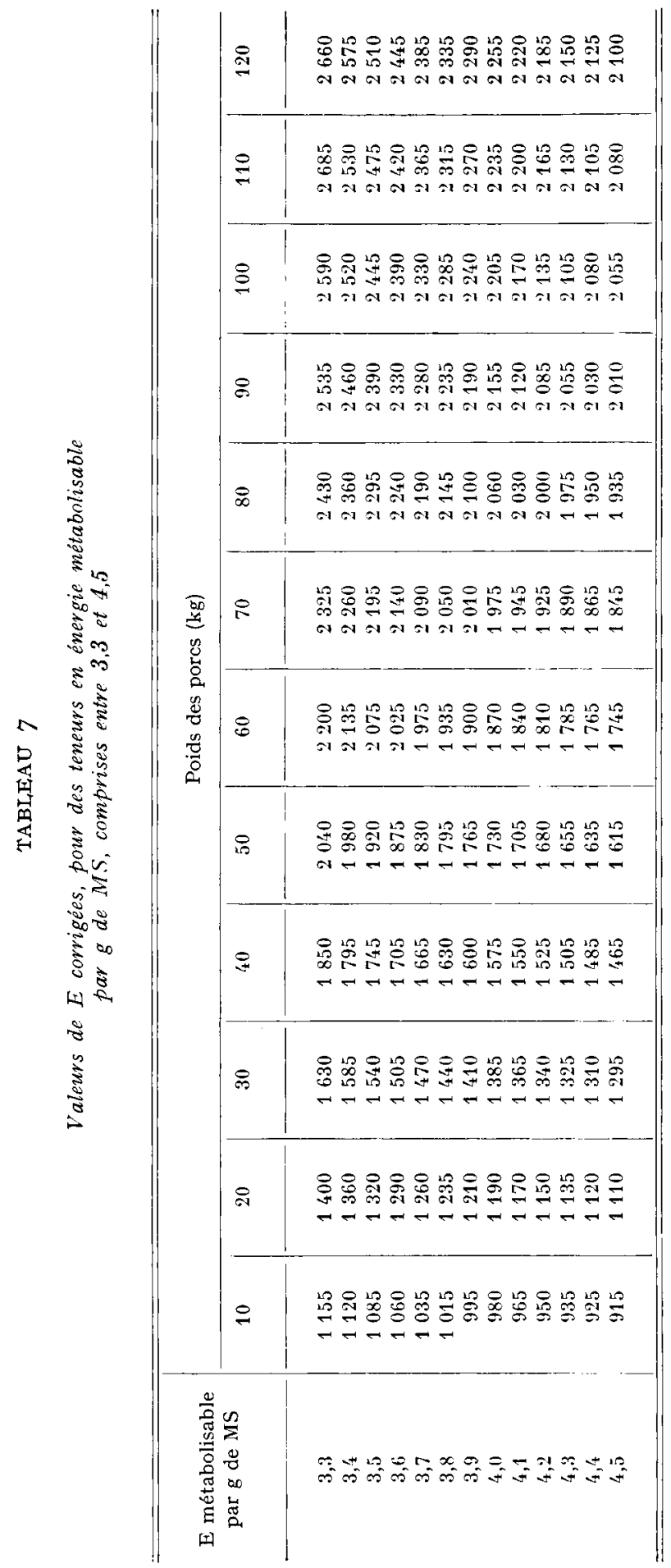


de la moyenne 3,9/g MS, nous avons calculé les données du tableau 7 qui nous montrent que les valeurs de $\mathrm{E}$ ne présentent que des corrections négligeables, pratiquement sans importance ( \pm 5 p. Ioo), lorsque l'apport d'énergie métabolisable par g de MS se maintient entre 3,7 et 4 , I kcal, ce qui est en général le cas pour la grande majorité des combinaisons d'aliments utilisés pour les porcs (tabl. 7).

Pour utiliser ce tableau, il convient de suivre les indications que voici :

I $^{\circ}$ Composer une formule alimentaire convenablement équilibrée en matières azotées et minérales, correctement adaptée à l'âge et au poids des animaux qu'il s'agit de nourrir.

$2^{\circ}$ Calculer avec l'aide des tables de composition des aliments la quantité d'énergie métabolisable provenant de la consommation d'un gramme de matière sèche de la ration prévue.

$3^{\circ}$ Déterminer d'après le tableau 8 le besoin $\mathrm{F}$ d'entretien correspondant aut poids des animaux.

L'étude des résultats individuels obtenus au cours des expériences qui nous: ont servi pour faire ce travail montre qu'il existe, d'un animal à l'autre, de notables différences en ce qui concerne les besoins d'énergie pour l'entretien. Si nous désignons par E le besoin d'entretien moyen d'un porc au cours des 5 ou 6 expériences de mesures énergétiques subies par lui, et par $\mathrm{E}_{m}$ le besoin moyen pour un même poids $\mathrm{P}$ calculé à l'aide des courbes de notre graphique, nous constatons que le rapport $\frac{\mathrm{E}}{\mathrm{E}_{m}}$ $\times$ Ioo varie d'un sujet à l'autre, et que pour certains d'entre eux, ce rapport peut varier de $t \pm \mathrm{r} 2 \mathrm{p}$. Ioo. Il existe donc bien des animaux qui utilisent mieux que les autres l'énergie métabolisable qu'ils reçoivent, et leur détection devrait permettre une sélection efficace à partir de cette particularité, si l'on parvenait à découvrir un moyen simple et suffisamment précis de la détecter. Or, il existe une liaison étroite entre le rapport $\frac{\mathrm{E}}{\mathrm{E}_{m}} \times$ roo, d'une part, et le coefficient moyen de digestibilité mesuré expérimentalement sur l'animal, d'autre part. Il suffit, pour s'en rendre compte, de se reporter aux données du tableau 8 qui ont été calculées avec l'aide de la documentation contenue dans la publication de NiELSEN (tabl. 8).

TABLEAU 8

Relation entre le rapport $\frac{E}{E_{m}} \times 100$ et le coefficient moyen de digestibilité

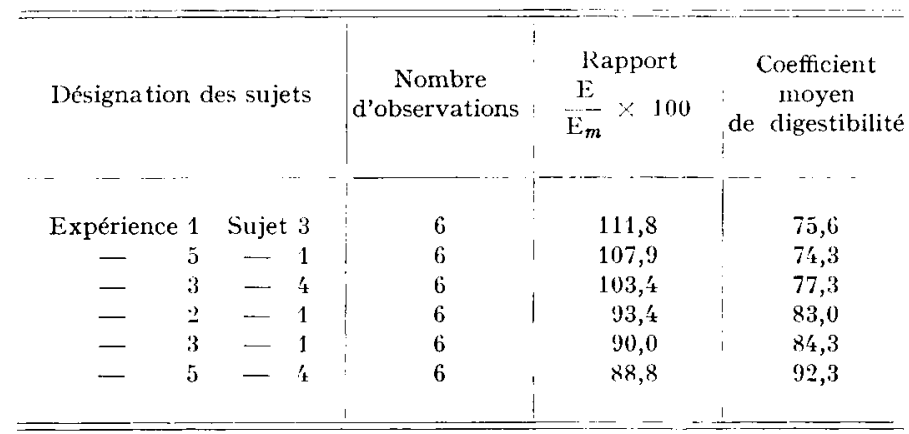


I1 serait utile de rechercher pour quelles raisons des animaux ayant fait l'objet d'une longue et patiente sélection, comme les porcs Landrace danois présentent entre eux de tels écarts en ce qui concerne l'utilisation digestive de leurs aliments. De toute façon, l'effet d'une digestibilité inférieure à la moyenne (8I,2 pour les r20 mesures de NIELSEN) entraîne fatalement pour les sujets possédant cette particularité une consommation plus grande de matière sèche alimentaire pour assurer l'équilibre de leur besoin d'entretien, ce qui est conforme à ce que nous savons de 'influence indéniable de la matière sèche ingérée sur la dépense d'énergie des animaux.

Reçu pour publication en juillet 1973.

\title{
SUMMARY
}

\section{DETERMINATION OF ENERGY REQUIREMENT \\ FOR MAINTENANCE IN THE PIG}

\begin{abstract}
The litterature gives several formulae allowing determination of the requirements for maintenance according to live weight. These formulae express the requirement either in the form of metabolizable energy or net energy. The results obtained by means of these two determination methods can easily be compared (see also Kielanowski) as, in the normally fed pig, the requirement for net energy represents $72 \mathrm{p}$. I oo of the metabolizable energy.

Results obtained by means of 5 different formulae, calculated in net energy, were compared in pigs between Io and $120 \mathrm{~kg}$. They appeared to be very different. $A$ great number of data reported in publications from Danish experimental stations, from I956 to 1970, allow, on the basis of energy balances, to state precisely the weights of the animals, the supplies of metabolizable energy, the energy retained corresponding to daily weight gains, the amounts of heat loss and the corresponding quantity of dry matter intake. It is then possible in each case to obtain the value $E$ of the requirement for maintenance by applying the relation : metabolizable energy $\mathrm{M}=$ energy for maintenance $\mathrm{E}+$ Energy for growth $\mathrm{P}+\mathrm{MS} \times \mathrm{I} \mathrm{kcal}$, in which MS represents the dry matter intake in $\mathrm{g}$. In this case, the ratio $\frac{\mathrm{M}-(\mathrm{E}+\mathrm{P})}{\mathrm{MS}}$ is admitted to be equal to $\mathrm{I}$.

From the results of $\mathrm{I} 64$ experiments carried out on 36 pigs between 20 and $\mathrm{I}$ oo $\mathrm{kg}$ by LUDvigSen and THorbek on the one hand (group A) and, on the other hand by Nielsen (group B), and on the basis of mean values of E calculated separately for each group, graphs were established, indicating for both cases the variation of $E$ (ordinate) as related to weight (abscissa). Two almost parallel curves were obtained separated by a distance equivalent to $150 \mathrm{kcal}$. This difference can easily be explained by the fact that the diets of NiELSEN were less digestible than those of LUDVIGSEN and THorbek. The food mixtures eaten by the animals of group A supplied 4.1 kcal metabolizable energy per $\mathrm{g}$, whereas those of group B only contained $3.65 \mathrm{kcal}$. To meet the requirements for maintenance, the animals of group $B$ therefore had to eat more dry matter than those of group A. For pigs weighing for instance $50 \mathrm{~kg}$, the difference of $5_{50} \mathrm{kcal}$ between the calculated $E$ values corresponded to a difference of $150 \mathrm{~g}$ in the dry matter intake. This observation gives an excellent proof of the rule relating heat increment above maintenance to the dry matter intake.
\end{abstract}

\section{RÉFÉRENCES BIBLIOGRAPHIQUES}

Breirem K., I939. Der Energieumsatz bei den Schweinen. Biedermanns Zentbl. Tierernähr, 11, $487-528$.

liotaRbinska M., KiElanowski J., I967. Energy balance studies with growing pigs by the comparative slaughter technique. EAAP, 4th Symposium on Energy metabolism, Varsovie, publ., 12, 299-310.

Kílelanowski J, I964. Estimates of the energy cost of protein deposition in growing animals. EAAP $3 r d$ Symposium on energy metabolism, Troon, publ. I I, I3-20. 
Kiblanowski J., Kotarbinska M., I970. In Energy metabolism of farm animals. EAAP 5th symposium onEnergy metabolism, Vitznau, publ. $\mathrm{x}_{3}$, I45-I48. Juris Druck + Verlag, Zurich.

Kíeiber M., r964. Metabolic body size. EAAP 3rd Symposium on Energy metabolism, Troon, Publ. I I, 427-435.

lekroy A. M., Charlet-Lery G., 1946. Expériences sur la croissance des porcelets avant et pendant le sevrage. Ann. Agron., 1, r-I7.

I.eroy A. M., I949. Norms for energetic feeding. C. R. Ve Congr. de Zootech., 53-80.

Leroy A. M., I954. Utilisation de l'énergie par les animaux. Ann. Zootech,, 4, 335-370.

I.EROY A. M., I964. Analysis of the origin of the heat loss of pigs fed normally. EAAP. 3rd Symposium on Energy metabolism, Troon, Publ. I1, 37-47.

Leroy A. M., I969. Idées nouvelles concernant l'utilisation de l'énergie par les animaux. C.R. A cad. Agr. de France, I9 nov. I969, II 33-II5I.

l.EROY A. M., I97r. Détermination de l'énergie métabolisable d'une ration pour porcs en fonction cless éléments digestibles. C. R. Acad. Agr. de France, 2 I avril 1971, 533-535.

Yielsen J., r970. The energy value of balanced fed rations for growing pigs determined by different methods. 381 Beretning fra Forsgoslaboratoriet, Copenhague, $2 \mathrm{I} 2 \mathrm{p}$.

I.vpvigsen J., Thorbek G., 1955. The effect of aureomycine on the metabolism of pigs and netabolic process in barrows and gilts. 283 Beretning fra Forsglaboratoriet, Copenhague.

lнorвек G., 1967. Studies of the energy metabolism of growing pigs. F.4.4P 4th symposium on inergy metabolism, Varsovie, publ. I2, 281-29o.

l'новвек G., I970. In Energy metabolism of farm animals. EAAP publ. 1:3, jth symposium on cner.' metabolism, I29-132, Juris Druck + Verlag, Zurich.

तiergaard L., Thorbek G., I972. Protein deposition and energy metabolism. IXth International Congress of Nutrition, Section C, Food for the expending world Mexico, sept. 1972. 